Rapid Reviews COVID-19

\title{
Review 1: "Inhibition of
}

SARS-CoV-2 infection in

human cardiomyocytes by

targeting the Sigma-1

receptor disrupts

cytoskeleton architecture

and contractility"

\section{Carmen Abate ${ }^{1}$}

${ }^{1}$ Università degli Studi di Bari

Published on: Mar 29, 2021

License: Creative Commons Attribution 4.0 International License (CC-BY 4.0). 


\section{$\underline{\text { RR:C19 Evidence Scale rating by reviewer: }}$}

- Reliable. The main study claims are generally justified by its methods and data. The results and conclusions are likely to be similar to the hypothetical ideal study. There are some minor caveats or limitations, but they would/do not change the major claims of the study. The study provides sufficient strength of evidence on its own that its main claims should be considered actionable, with some room for future revision.

$* * * * * * * * * * * * * * * * * * * * * * * * * * * * * * * * * * * * * * *$

\section{Review:}

The manuscript by Salerno et al deals with the anti-SARS-CoV-2 action of a reference sigma-1 receptor antagonist, NE100, in human cardiomyocytes. In particular, it shows how, in these cells, NE100 reduces SARS-CoV-2 infection and viral replication, reduces cell death associated with infection, and attenuates the release of inflammatory cytokines (IL6 and IL8). However, contractility is importantly reduced, likely because of a verified disruption of the cytoskeleton architecture.

The paper is very well structured, supported by evidence, and in my opinion deserves to be published. Nevertheless, another sigma-1 receptor reference compound should be used, in order to more confidently correlate the effects we see with the interaction of NE100 with sigma-1 receptors, at least in a couple of assays that the authors consider more representative. Alternatively, experiments with sigma-1 receptors KO cells could be conducted. This is particularly important as recent literature, that has to be cited (Gordon et al, Science 2020, 370, 1181) because of its breakthrough findings, shows how the $\mathrm{KO}$ and $\mathrm{KD}$ of sigma-1 receptors drastically reduce SARS-CoV-2 infection. However, no clear correlation between sigma-1 receptor affinity of the ligands tested and antiviral activity is observed in the cited paper. While unraveling sigma-1 receptors as crucial host dependency factors for the virus infection, the paper by Gordon et al. also points out as the effect of sigma ligands has to be extensively studied, and can go beyond the definition of sigma-1 agonist and antagonist. Therefore, it is of crucial importance to further support that NE100 antiviral action is mediated by sigma-1 receptor, in order to confidently direct research efforts towards investigations of the sigma-1 receptors as a druggable target against the coronavirus responsible for the COVID-19 pandemic (and likely other viruses). 
Overall, results presented in this manuscript support this role of sigma-1 receptor (but can strengthen it more) and provide an important alert about the toxic cardiac effects that sigma-1 ligands, such as NE100, may have, while also providing a convenient model to study these cardiac effects. 